\title{
Load Flow and Transient Stability Analyses for an Integrated Solar Combined Cycle Station in Iraqi Southern by Using ETAP
}

\author{
Ahmed Z. Abass*, Dmitry A. Pavlyuchenko, \\ Alexandr V. Prokopov and Saadallah Hussain Zozan \\ Novosibirsk State Technical University \\ Novosibirsk, Russian Federation
}

Received 10.07.2020, received in revised form 21.07.2020, accepted 06.02.2021

\begin{abstract}
Analyses of a power system are important for designing and operating phase execution monitoring and to ensure reliable grid operations by sufficient protection projects settings. In this paper, electrical model of a $340 \mathrm{MW}$ integrated solar combined cycle (ISCC) located in the south of Iraq, is developed by Electrical Transient Analyzer Program (ETAP) and load flow, voltage stability and short circuit analyses are performed. Impact of power grid voltage instability on system buses of the power plant is estimated. Using load flow analysis that use Newton-Raphson algorithm, buses operating at under voltage due to power grid voltage instability are specified and their voltages are improved according to given voltage limitation that are based on buses criticality with regard to loads. On-load tap changers and reactive power compensation are used to improve steady state voltage stability. Optimal position for capacitor banks placement and number of capacitor banks are proposed by using optimal capacitor placement module of ETAP. The use of modern technology and advance planning have a big impact on reducing losses. The article shows that the lack of planning is one of the main causes of energy losses. The parameters of the module also indicate the voltage limits, bus voltage and ratings of available capacitor banks. The voltage limit is set at $95 \% \leq \mathrm{V} \leq 110 \%$, and it is global for all buses.
\end{abstract}

Keywords: load flow, ETAP, Integrated Solar Combined Cycle (ISCC), transient stability, optimal capacitor placement(OCP), electric power systems, reactive power compensation, low voltage problem.

Citation: Abass A.Z., Pavlyuchenko D.A., Prokopov A.V., Zozan S.H. Load flow and transient stability analyses for an integrated solar combined cycle station in iraqi southern by using ETAP, J. Sib. Fed. Univ. Eng. \& Technol., 2021, 14(1), 5-16. DOI: $10.17516 / 1999-494 X-0285$

(C) Siberian Federal University. All rights reserved

This work is licensed under a Creative Commons Attribution-Non Commercial 4.0 International License (CC BY-NC 4.0).

* Corresponding author E-mail address: theking.amir@mail.ru, saspro684@gmail.com

ORCID: 0000-0003-1836-3435 (A. Abass); 0000-0003-4522-9557 (Pavlyuchenko D.); 0000-0001-8948-2277 (Hussain Z.) 


\title{
Анализ потокораспределения и устойчивости для электрической системы с электростанцией с комбинированным циклом в Южном Ираке с использованием ЕТАР
}

\author{
А. З. Абасс, Д. А. Павлюченко, \\ А. В. Прокопов, 3.С. Хуссейн \\ Новосибирский государственный технический университет \\ Российская Федерация, Новосибирск
}

\begin{abstract}
Аннотация. Анализ потокораспределения, устойчивости и токов короткого замыкания важен на этапе как проектирования, так и эксплуатации электроэнергетических систем (ЭЭС). В работе представлена модель электрической системы с электростанцией с комбинированным циклом Integrated Solar Combined Cycle (ISCC) мощностью 340 МВт в Южном Ираке, разработанная в программе анализа электрических переходных процессов Electrical Transient Analyzer Program (ETAP), также, помимо этого, произведен расчет режимов. Оценивается влияние нестабильности напряжения в энергосистеме на шинах электростанции. Используя расчетный модуль ЕТАР на основе метод Ньютона-Рафсона, определяем шины, работающие на пониженном напряжении из-за нестабильности в системе. Для повышения стабильности в системе использованы устройства регулирования напряжения трансформаторов под нагрузкой (РПН) и компенсация реактивной мощности. Оптимальное размещение конденсаторных батарей и значения мощности конденсаторов выбрано с применением встроенного модуля ЕТАР. Использование современных технологий и заблаговременное планирование оказывают большое влияние на снижение потерь. В статье показано, что отсутствие планирования является одной из основных причин потерь электроэнергии. В параметрах модуля указаны также ограничения по напряжению, напряжение на шинах и номиналы доступных конденсаторных батарей. Ограничение напряжения установлено на уровне $95 \% \leq \mathrm{V} \leq 110 \%$, и оно является глобальным для всех шин.
\end{abstract}

Ключевые слова: анализ потокораспределения, устойчивость, электростанция с комбинированным циклом, ток короткого замыкания, оптимальное размещение конденсаторных батарей, ЕТАР, электроэнергетические системы (ЭЭС), понижение напряжения, компенсация реактивной мощности.

Цитирование: Абасс, А.З. Анализ потокораспределения и устойчивости для электрической системы с электростанцией с комбинированным циклом в Южном Ираке с использованием ЕТАР / А. З. Абасс, Д. А. Павлюченко, А. В. Прокопов, 3. С. Хуссейн // Журн. Сиб. федер. ун-та. Техника и технологии, 2021, 14(1). С. 5-16. DOI: 10.17516/1999-494X-0285

\section{Введение}

С 1980 г. по сегодняшний день Ирак ведет непрерывную войну, которая уничтожила всю инфраструктуру. В 1980 г. началась Ирано-Иракская война, которая продолжалась восемь лет. Это вызвало увеличение государственных расходов на войну и сократило расходы на развитие инфраструктуры, особенно на электроэнергетику. В 2020 г. Ирак по-прежнему страдал от острой нехватки электроэнергии для граждан. Отмечается, что 90 \% заводов и муниципальных зданий все еще не работают. Спрос на электроэнергию в Ираке в 2007 г. составлял 11000 МВт, в 2013 г. 16000 и летом 2018 г. - 24500 . Ожидается, что этот спрос увеличится до более чем 30000 МВт в 2022 г. [1, 2, 3].

Около 70 \% всей электроэнергии, произведенной в 2013 г., включая технические, коммерческие и административные потери (передача $-6 \%$, распределение - $13 \%$; воровство и не- 
оплаченные счета - $23 \%$; несобранные - $26 \%$; собранные - 33 \%), было потеряно. Более $90 \%$ потерь приходится на распределительную сеть, из которых 79 \% - нетехнические потери (воровство, неоплаченные и несобранные счета) [3]. Распределительная система в целом находится в плохом состоянии и, по-видимому, является одной из проблем в электроснабжении, поскольку она деградирует из-за неэффективного управления и отсутствия инвестиций. Неконтролируемое и незапланированное расширение, в дополнение к старению сети, привело к перегрузкам и большим техническим и нетехническим потерям. Например, распределительные линии, которые несут ограниченное количество электроэнергии на короткие расстояния, являются основным фактором потерь в системе. Общие потери при передаче и распределении составляют около 40-50 \% системных потерь в Ираке, причем большая часть этих потерь обусловлена сектором распределения (т. е. 80-90 \% от общих потерь при передаче и распределении) $[4,5]$. Неэффективное управление и эксплуатация сектора распределения способствуют этой проблеме, включая выставление счетов, учет, обслуживание клиентов и невозможность повысить производительность системы. Использование современных технологий и заблаговременное планирование оказывают большое влияние на снижение потерь. В статье показано, что отсутствие планирования является одной из основных причин потерь электроэнергии. Потокораспределение, стабильность напряжения и анализ токов короткого замыкания всегда нужны для правильной установки, стабильной и надежной работы электроэнергетической системы. Этот анализ дает информацию, которая может потребоваться для дальнейшего расширения и повышения эффективности. Анализ потокораспределения - математический подход и инструмент, который используется инженерами-электриками для планирования и определения различного напряжения на шинах, их фаз, активных и реактивных мощностей.

Наиболее часто используются итерационные методы при решении задачи потокораспределения энергии: Ньютона-Рафсона, Гаусс Зайделя и др. В связи с быстрой сходимостью и меньшим количеством итераций метод Ньютона-Рафсона по сравнению с другими методами более эффективен.

Стабильностью напряжения энергосистемы является ее способность поддерживать допустимое напряжение на всех своих шинах при возмущениях в системе. Это зависит от способности поддерживать равновесие между нагрузкой и генерацией. Понижение напряжения, вызванное возмущениями, негативно влияет на систему распределения.

Нестабильность вызывает постепенное повышение или, наоборот, падение напряжения на некоторых шинах, что приводит к отключению нагрузок, линий электропередачи и других элементов системы, вследствие чего из-за этих отключений некоторые генераторы могут выйти из синхронизма.

Регулирование реактивной мощности широко используемый метод для повышения стабильности напряжения системы. Оптимальное значение для реактивной мощности установки играет жизненно важную роль в определении улучшения стабильности напряжения. Компенсация реактивной мощности на «слабых» шинах в целом улучшает стабильность напряжения. В работе проведено моделирование электрической системы ISCC340 MBт в программном комплексе ЕТАР. После изменения напряжения узлы нагрузки идентифицируются и их напряжения улучшаются в соответствии с желаемым диапазоном напряжения при помощи настройки РПН и оптимального размещения конденсаторов $[6,7]$. 


\section{Описание объекта}

Объектом исследования является электрическая система ISCC на 340 MBт с четырьмя газотурбинными и двумя паротурбинными установками. Четыре газотурбинных генератора мощностью 50 МВт каждый и два паротурбинных генератора мощностью 65 и 75 МВт подключены к изолированным шинам Khur Zubair. Каждый генератор подключен к центральной шине с помощью повышающего трансформатора и вырабатывает энергию на напряжении 20 кВ, затем преобразует в 132 кВ, чтобы отправить полученную мощность в общую электрическую сеть [8-10].

Два вспомогательных трансформатора с тремя обмотками и мощностью 250 МВА используются для понижения напряжения с 400 до 132 кВ общей сети и до 11 кВ для собственных нужд электростанции, а также для питания небольших жилых районов. Шина Khur Zubair питает восемь основных нагрузок (сталелитейный завод - 22 МВт, Фао - 21,6 MBт, Bab Zubair 20,5 МВт, месторождение «Румайла» - 80 МВт, город Ум-Касер и порт - 220 МВт, месторождение «Zubair» - 45 МВт, порт Альбакер - 20 МВт, центр города - 110 МВт). Большинство нагрузок потребляется на нефтяных месторождениях, фабриках и в портах, так как дефицит покрывается за счет общей сети. Модель данной системы разработана в ЕTAР (рис. 1a, 1b), а затем выполнены необходимые расчеты, как указано в дальнейшем.

\section{Анализ потокораспределения}

Анализ потокораспределения является инструментом и математическим подходом, применяемыми инженерами-электриками для определения параметров электрической системы при нормальной устойчивой работе, что очень полезно на этапе планирования и эксплуатации. Чтобы выполнить анализ потокораспределения, сначала необходимо построить матрицу узловых проводимостей $\left(\mathrm{Y}_{\mathrm{bus}}\right)$. Используя $\mathrm{Y}_{\text {bus }}$, можно записать узловые уравнения для электрической системы следующим образом [6, 11-13]:

$$
I=Y_{b u s} * U
$$

где I, $\mathrm{Y}_{\text {bus }}$ и $\mathrm{U}$ - ток, узловая проводимость и напряжение соответственно.

Узловое уравнение в обобщенном виде для системы шин n:

$$
I_{i}=\sum_{j=1}^{n} Y_{i j}^{*} * U_{j}
$$

где $\mathrm{i}=1,2,3, \ldots \mathrm{n}$.

Полная мощность, подводимая к узлу i,

$$
\begin{aligned}
& P_{i}+j Q_{i}=U_{i} I_{i}^{*}, \\
& I_{i}=\frac{P_{i}-j Q_{i}}{U_{i}^{*}},
\end{aligned}
$$

где $\mathrm{P}_{\mathrm{i}}$ и $\mathrm{Q}_{\mathrm{i}}$ - активная и реактивная мощность в узле $\mathrm{i}$.

Подставляя $\mathrm{I}_{\mathrm{i}}$ через $\mathrm{P}_{\mathrm{i}}$ и $\mathrm{Q}_{\mathrm{i}}$, получаем

$$
\frac{P_{i}-j Q_{i}}{U_{i}^{*}}=U_{i} \sum_{j=1}^{n} Y_{i j}-\sum_{j=1}^{n} Y_{i j} U_{j}
$$

где $\mathrm{j} \neq \mathrm{i}$. 


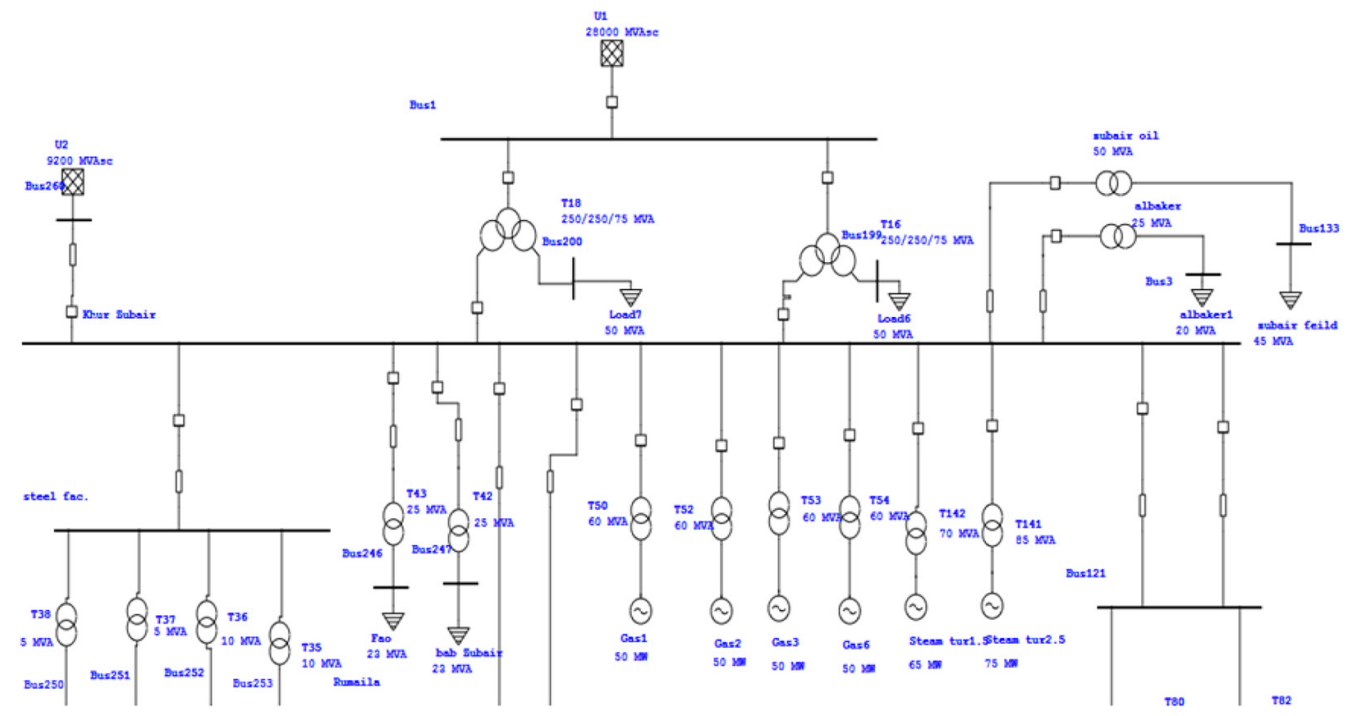

Рис. 1а. Модель однолинейной схемы электрической системы с электростанцией с комбинированным циклом мощностью 340 МВт

Fig .1a. Model of a single-line diagram of an electrical system with a combined cycle power plant with a capacity of $340 \mathrm{MW}$

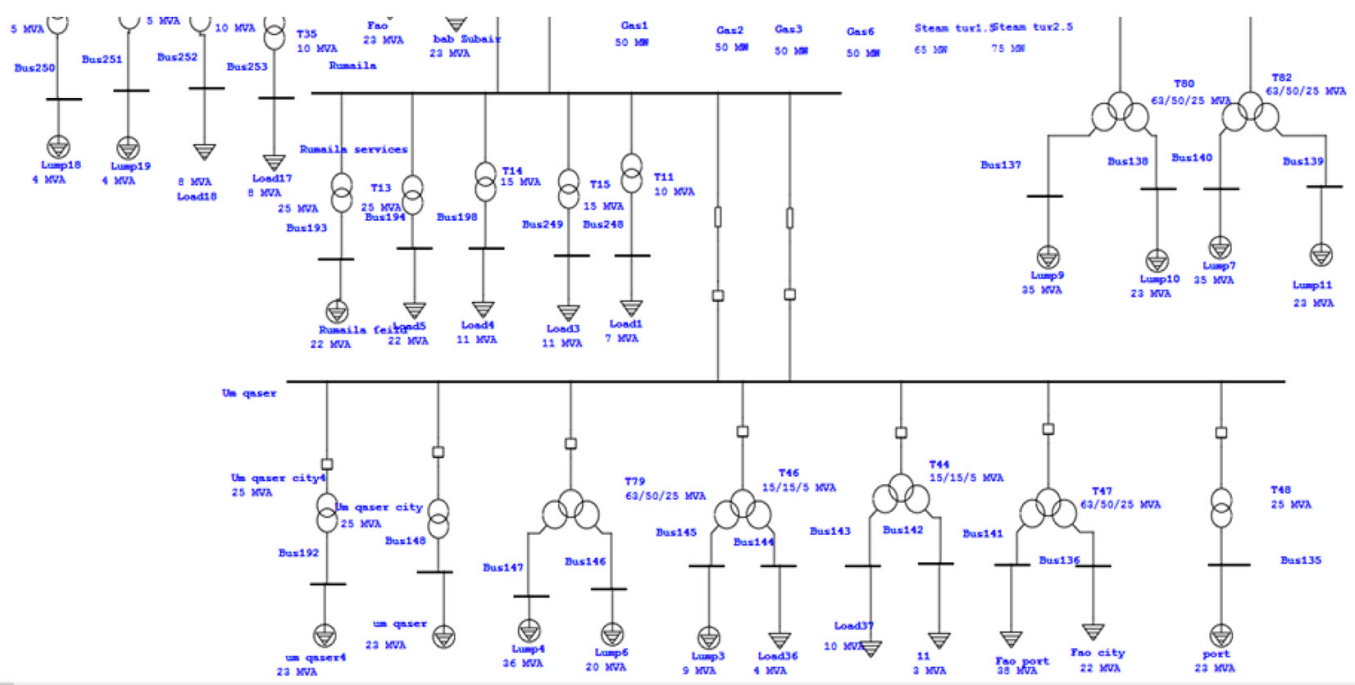

Рис. 1b. Модель однолинейной схемы электрической системы с электростанцией с комбинированным циклом мощностью 340 МВт

Fig. 1b. Model of a single-line diagram of an electrical system with a combined cycle power plant with a capacity of $340 \mathrm{MW}$

Различные методы расчета потокораспределения применяются для анализа устойчивого состояния электрической системы. В работе используется алгоритм Ньютона-Рафсона по причине более быстрой сходимости и наименьшего количества итераций по сравнению с другими методами при выражении уравнения (2) в полярной форме и подставлении его в уравнение 
(3), действительные и мнимые составляющие результирующего уравнения будут иметь вид

$$
\begin{aligned}
& P_{i}=\sum_{j=1}^{n}\left|U_{i}\right|\left|U_{j}\right|\left|U_{i j}\right| \cos \left(\theta_{i j}-\delta_{i}+\delta_{j}\right), \\
& Q_{i}=\sum_{j=1}^{n}\left|U_{i}\right|\left|U_{j}\right|\left|U_{i j}\right| \sin \left(\theta_{i j}-\delta_{i}+\delta_{j}\right)
\end{aligned}
$$

где $\delta_{\mathrm{i}}, \delta_{\mathrm{i}}$ и $\theta_{\mathrm{ij}}-$ углы $\mathrm{U}_{\mathrm{i}}, \mathrm{U}_{\mathrm{j}}$ и $\mathrm{U}_{\mathrm{ij}}$ соответственно.

Уравнения (6) и (7) разложены в ряды Тейлора относительно начальной оценки, пренебрегая всеми членами более высокого порядка, запишем их в матричной форме $[11,14,16]$ :

$$
\left[\begin{array}{l}
\Delta P \\
\Delta Q
\end{array}\right]=\left[\begin{array}{ll}
J_{1} & J_{3} \\
J_{2} & J_{4}
\end{array}\right]\left[\begin{array}{c}
\Delta \delta \\
\Delta|V|
\end{array}\right]
$$

где $\mathrm{J}_{1}-\mathrm{J}_{4}$ являются элементами матрицы Якоби.

Диагональные и недиагональные элементы $\mathrm{J}_{1}$ :

$$
\begin{aligned}
& \frac{\partial P i}{\partial \delta i}=\sum_{j \neq 1}\left|U_{i}\right|\left|U_{j}\right|\left|U_{i j}\right| \cos (\theta i j-\delta i+\delta j) \\
& \frac{\partial P i}{\partial \delta i}=-\left|U_{i}\right|\left|U_{j}\right|\left|U_{i j}\right| \sin (\theta i j-\delta i+\delta j)
\end{aligned}
$$

Разница между графиком и расчетными значениями мощности, известными как невязки мощности для членов $\Delta P_{i}^{(k)}$ и $\Delta Q_{i}^{(k)}$, такова:

$$
\begin{aligned}
& \Delta P_{i}^{(k)}=\Delta P_{i}^{s c h}-P_{i}^{(k)}, \\
& \Delta Q_{i}^{(k)}=\Delta Q_{i}^{s c h}-Q_{i}^{(k)} .
\end{aligned}
$$

Новые оценки напряжения на шине:

$$
\begin{aligned}
& \delta^{(k+1)}=\delta_{i}^{(k)}+\Delta \delta_{i}^{(k)}, \\
& \left|U^{k+1}\right|=\left|U_{i}^{(k)}\right| \Delta\left|U_{i}^{(k)}\right| .
\end{aligned}
$$

После проведения анализа потокораспределения с использованием ЕТАР генерируется сводный отчет о предупреждениях, который сообщает нам, какая часть системы требует немедленного внимания. На рис. 2a, 2b ясно видно, что Umqaser и шина 121 работают на нижнем напряжении и эти шины содержат множество ответвлений с номинальным напряжением (11 и 33 кВ). В табл. 1 приведены следующие данные: выработка электроэнергии генераторами, потребляемая мощность нагрузок и передаваемая мощность.

\section{Анализ устойчивости}

Определяется реакция системы на различные помехи, которые являются источником нестабильности, т. е. приводят к потере синхронности, остановке или перегрузке генераторов и двигателей.

Переходные процессы в основном связаны с неисправностью коммутационных аппаратов, переключением конденсаторных батарей и другими часто переключаемыми нагрузками $[7,11]$. В этой статье демонстрируется устойчивость системы, когда она теряет одну из своих нагрузок 


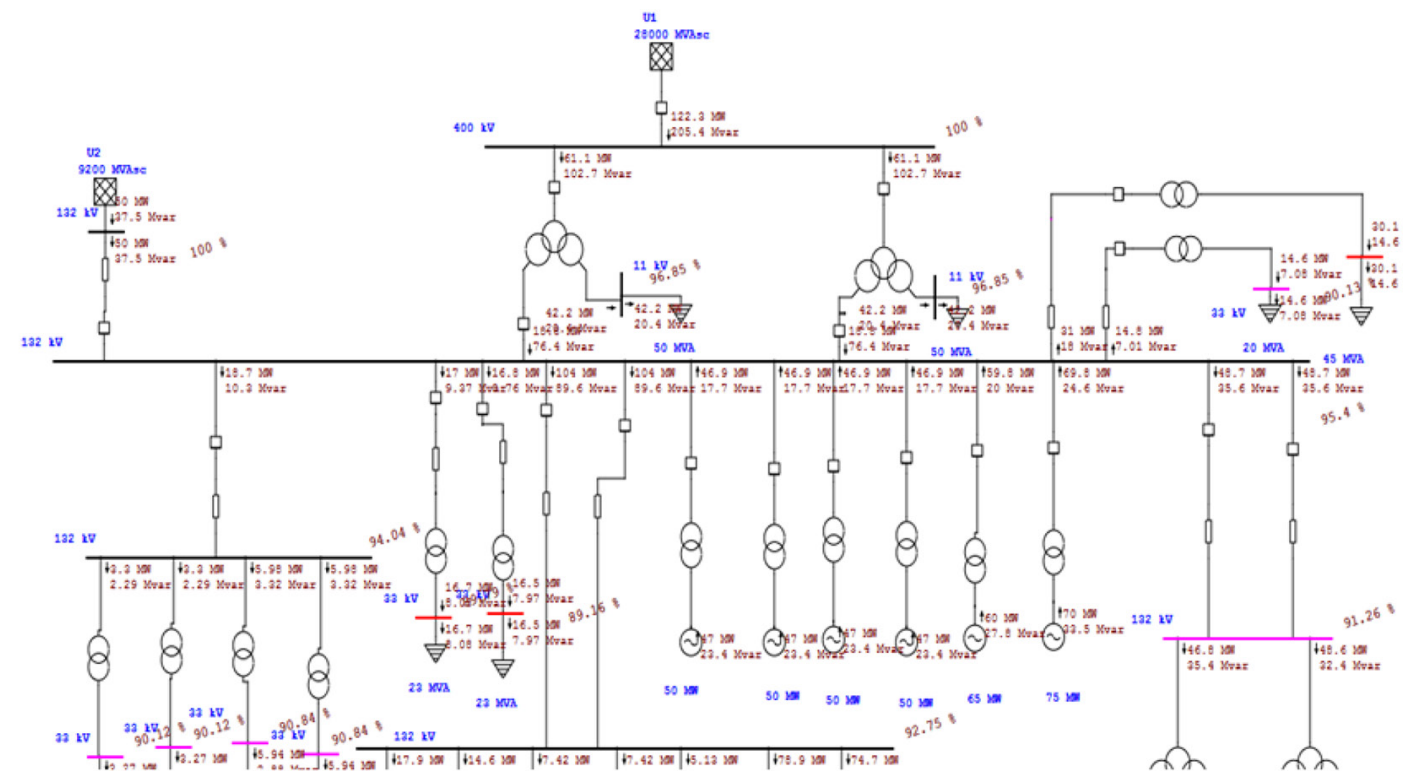

Рис. 2а. Результаты расчета потокораспределения

Fig. 2a. Results of load flow

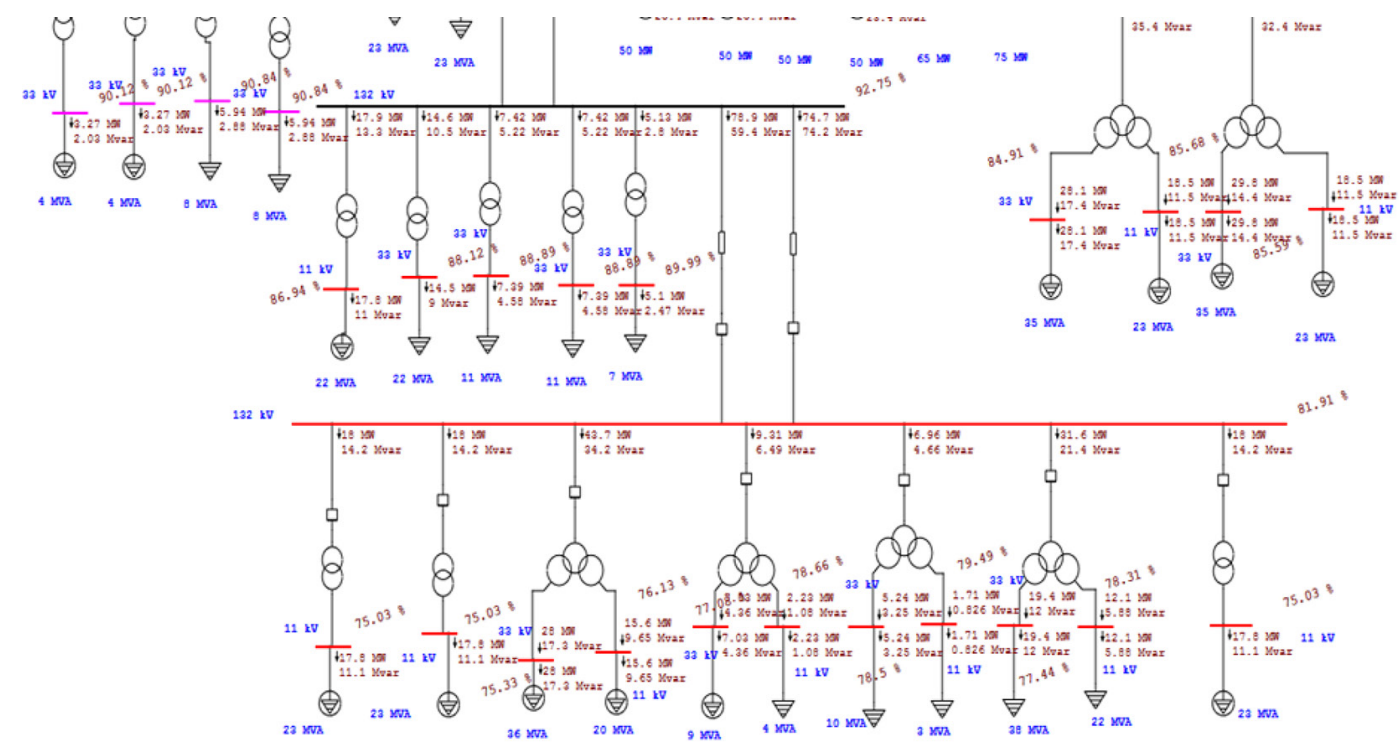

Рис. 2b. Результаты расчета потокораспределения

Fig. 2b. Results of load flow 
Таблица 1. Результаты анализа потокораспределения

Table 1. Results of load flow analyses

\begin{tabular}{|c|c|c|c|c|c|c|c|c|c|}
\hline \multirow{2}{*}{$\begin{array}{c}\text { Номер } \\
\text { узла }\end{array}$} & \multicolumn{3}{|c|}{ Напряжение } & \multirow{2}{*}{$\begin{array}{c}\text { Номер } \\
\text { ветви }\end{array}$} & \multirow[b]{2}{*}{$\mathrm{U}, \kappa \mathrm{B}$} & \multicolumn{4}{|c|}{ Параметры } \\
\hline & кВ & $\%$ & Угол & & & $\begin{array}{c}\mathrm{P}, \\
\mathrm{MBT}\end{array}$ & Q, MBap & $\begin{array}{l}\mathrm{I}, \\
\mathrm{A}\end{array}$ & $\cos \varphi$ \\
\hline \multirow[t]{2}{*}{121} & 132 & 91,044 & $-3,9$ & $137 ; 138$ & $33 ; 11$ & 48,899 & 37,816 & 300,4 & 79,2 \\
\hline & & & & $139 ; 140$ & $11 ; 33$ & 51,624 & 35,270 & 304,5 & 82,7 \\
\hline \multirow[t]{11}{*}{ Umqaser } & 132 & 85,138 & $-6,2$ & 135 & 11 & 18,558 & 11,501 & 1326,5 & 85,3 \\
\hline & & & & 148 & 11 & 16,065 & 9,956 & 1165,0 & 85,4 \\
\hline & & & & 192 & 11 & 18,480 & 11,453 & 1338,9 & 85,6 \\
\hline & & & & 142 & 11 & 2,442 & 1,183 & 172,9 & 90 \\
\hline & & & & 143 & 33 & 5,301 & 3,285 & 131 & 85 \\
\hline & & & & 144 & 11 & 2,474 & 1,198 & 174,0 & 90 \\
\hline & & & & 145 & 33 & 7,196 & 4,459 & 176,6 & 85 \\
\hline & & & & 136 & 11 & 14,952 & 7,242 & 1003,5 & 90 \\
\hline & & & & 141 & 33 & 23,137 & 14,339 & 540 & 85 \\
\hline & & & & 146 & 11 & 16,085 & 9,969 & 1161,8 & 85 \\
\hline & & & & 147 & 33 & 26,682 & 16,536 & 631,6 & 85 \\
\hline
\end{tabular}

(поле Румайла 22 MBA). На рис. 3 и 4 показан угол мощности системы и частота вращения генераторов соответственно.

\section{Оптимальное размещение конденсаторных батарей}

Для оптимального размещения конденсаторных батарей в расчетном модуле ЕТАР применяется генетический алгоритм. Это процедура оптимизации, в которой используется философия естественного отбора. Целевая функция, включенная в этот модуль ЕТАР, имеет вид

$$
M_{\text {in }}=\sum_{i=1}^{N_{\text {bus }}}\left(X_{i} C_{0 i}+Q_{c i} C_{1 i}+B_{i} C_{2 i} T\right)+C_{2} \sum_{i=1}^{N_{\text {load }}} T l P_{L}^{l},
$$

где $\mathrm{N}_{\text {bus }}-$ число шин; $\mathrm{x}_{\mathrm{i}}-0 / 1,0$ означает, что на шине і установлен конденсатор; $\mathrm{C}_{0 \mathrm{i}}-$ стоимость установки; $\mathrm{C}_{1 \mathrm{i}}$ - стоимость конденсатора за кВар; $\mathrm{C}_{2 \mathrm{i}}$ - эксплуатационные расходы; $\mathrm{B}_{\mathrm{i}}-$ количество батарей конденсаторов; $\mathrm{Q}_{\mathrm{ci}}$ - конденсатор, размером батареи на кВар; Т - период планирования, в годах; $\mathrm{C}_{2}-$ стоимость потерь за кВтч; $l$ - уровни нагрузки; $\mathrm{T} l$ - продолжительность времени нагрузки L, в часах; $P_{L}^{l}$ - общие потери системы на уровне L.

В рассматриваемой задаче в электрической сети расчетный модуль определения оптимальных узлов для размещения конденсаторных батарей дает информацию о количестве конденсаторных батарей и местах их размещения, необходимых для реактивной компенсации и повышения напряжения указанных шин [6, 7, 12, 13, 16-18].

В целом при текущем уровне нагрузок и потокораспределении в электрической системе наблюдается понижение напряжения, для улучшения уровня которого используются устройства РПН трансформаторов $[6,7,12,13,18]$. Но так как их диапазона регулирования недостаточно, напряжение на некоторых шинах остается ниже 95 \%. Чтобы улучшить его, в дальнейшем ис- 


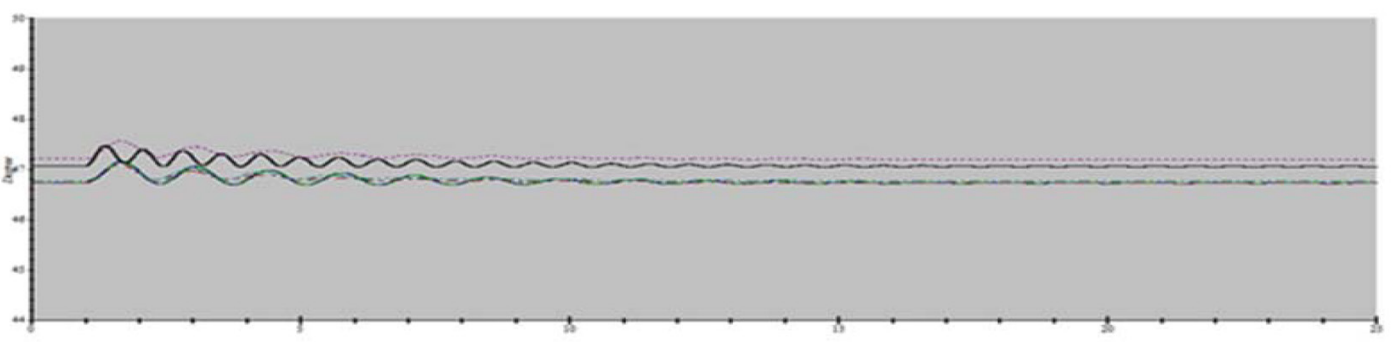

Рис. 3. Угол мощности системы

Fig. 3. Power angle for generators

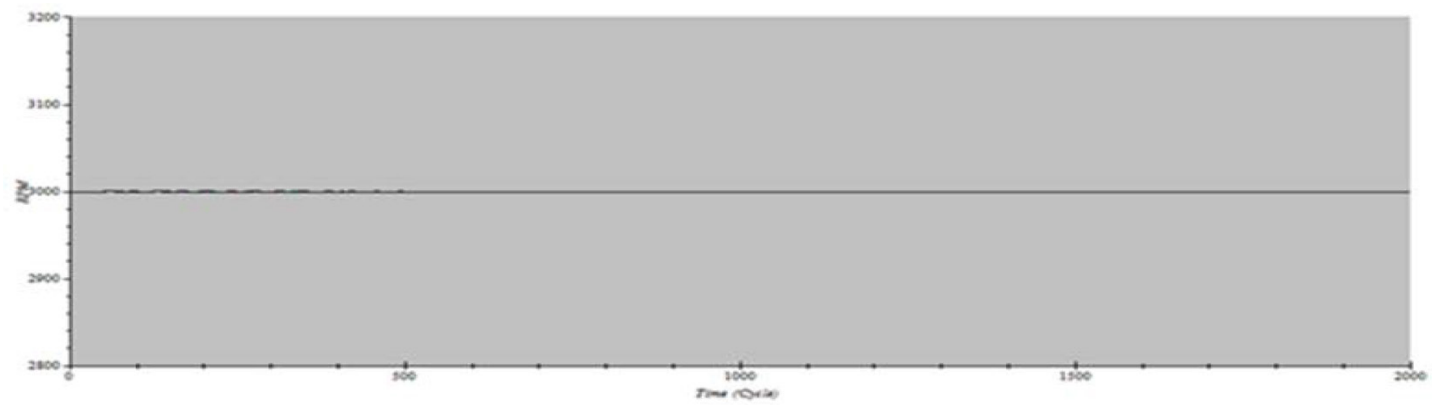

Рис. 4. Частота вращения генераторов

Fig. 4. Generators speed

пользуется модуль оптимального размещения конденсаторных батарей программного комплекса ETAP. В параметрах модуля указываются также ограничения по напряжению, напряжение на шинах и номиналы доступных конденсаторных батарей. Ограничение напряжения установлено на уровне $95 \% \leq \mathrm{V} \leq 110 \%$, и оно является глобальным для всех шин.

Результаты работы модуля оптимального размещения конденсаторных батарей приведены в табл. 2 и на рис. 5. Значения рабочего напряжения показывают, что напряжение обеих шин увеличивается в соответствии с установленным ограничением напряжения. Также указаны номинальные напряжения, размеры и количество конденсаторных батарей для каждой шины низшего напряжения. Сравнение напряжений, мощности и коэффициента мощности для шин Umqaser и 121 приведено в табл. 3.

\section{Выводы}

В работе рассматривается вопрос анализа потокораспределения и устойчивости электрической системы с использованием программного комплекса ЕТАР. Исследования потокораспределения важны для планирования будущего расширения энергосистем, а также для определения наилучшей работы существующих систем. Объектом исследования является фрагмент электрической системы Южного Ирака с электростанцией с комбинированным циклом. Рассмотренная электростанция предлагается для модернизации путем добавления комбинированного цикла для существующей газовой станции на юге Ирака в городе Басра. Так как он считается промыш- 


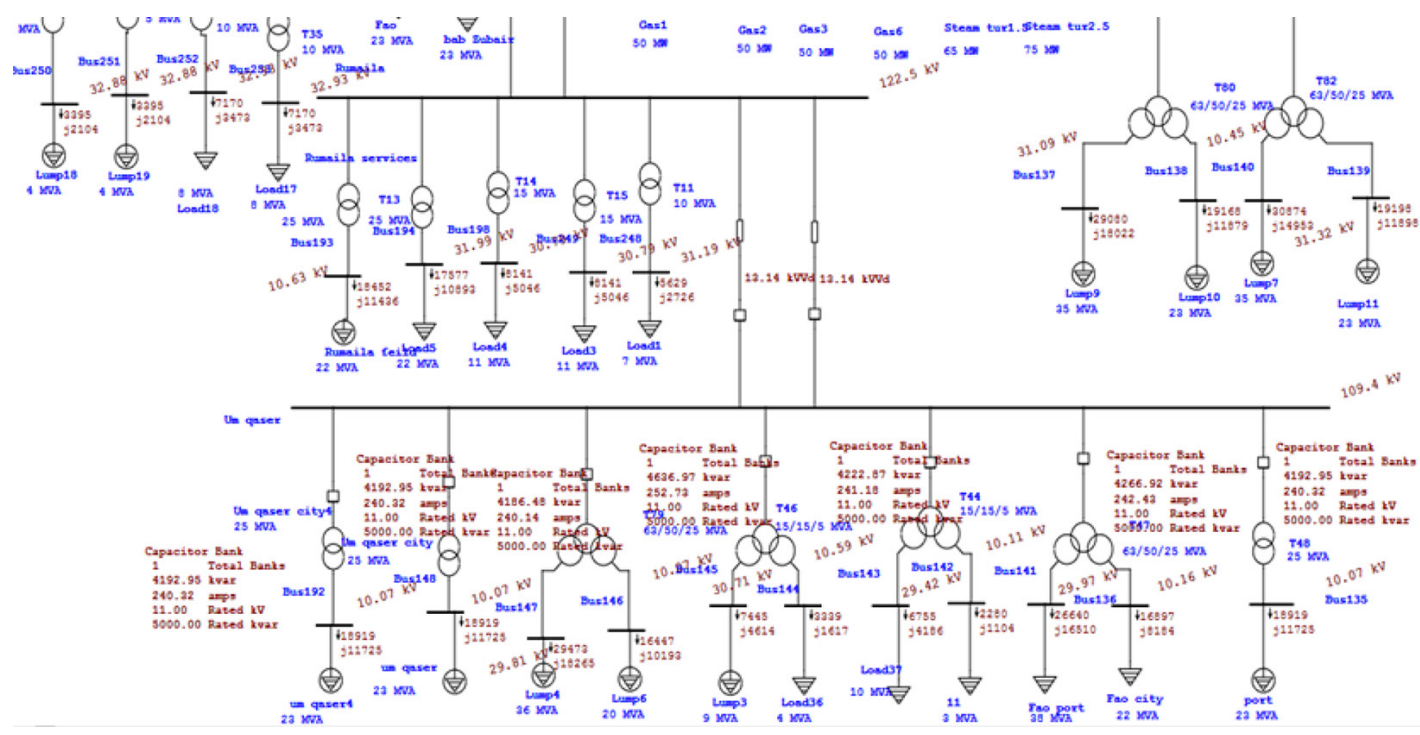

Рис. 5. Результаты после размещения конденсаторных батарей

Fig. 5. Results after OCP

Таблица 2. Результаты после размещения конденсаторных батарей

Table 2. Results after OCP

\begin{tabular}{|c|c|c|c|c|c|c|c|}
\hline \multirow{2}{*}{$\begin{array}{c}\text { Номер } \\
\text { узла }\end{array}$} & \multirow{2}{*}{$\begin{array}{c}\text { Номинальное } \\
\text { напряжение, кВ }\end{array}$} & $\%$ & угол & $\cos \varphi$ & \multicolumn{3}{|c|}{ Конденсаторные батареи } \\
\cline { 7 - 8 } & & & & & Кол-во & $\begin{array}{c}\text { Рабочая } \\
\text { мощность, кВар }\end{array}$ & $\begin{array}{c}\text { Номинальная } \\
\text { мощность, кВар }\end{array}$ \\
\hline 135 & 11 & 92,55 & $-16,35$ & 93,4 & 1 & 4281 & 5000 \\
\hline 136 & 11 & 91,695 & $-17,47$ & 93 & 1 & 4204 & 5000 \\
\hline 138 & 11 & 91,327 & $-10,5$ & 93 & 1 & - & 5000 \\
\hline 139 & 11 & 91,818 & $-10,52$ & 93 & 1 & - & 5000 \\
\hline 142 & 11 & 90,014 & $-16,60$ & 93 & 1 & 4051 & 5000 \\
\hline 144 & 11 & 92,528 & $-17,64$ & 93 & 1 & 4131 & 5000 \\
\hline 146 & 11 & 90,899 & $-17,53$ & 93 & 1 & 4280 & 5000 \\
\hline 148 & 11 & 91,066 & $-15,34$ & 93,8 & 1 & 4146 & 5000 \\
\hline 192 & 11 & 91,280 & $-16,10$ & 93 & 1 & 4195 & 5000 \\
\hline 193 & 11 & 96,269 & $-10,25$ & 90 & 1 & - & 5000 \\
\hline
\end{tabular}

ленным и густонаселенным городом, электрическая сеть страдает от постоянных отключений, больших потерь энергии и значительных нарушений напряжения, вызванных несколькими факторами, одним из которых является дефицит электрической энергии. С помощью программы ЕTAP разрабатывается решение этих проблем, а с помощью модуля оптимального размещения конденсаторных батарей были выявлены слабые узлы и добавлена группа конденсаторов для компенсации реактивной мощности. Показаны положительные результаты, полученные после работы с помощью модуля оптимального размещения конденсаторных батарей, вследствие 
Таблица 3. Параметры узлов

Table 3. Options of buses

\begin{tabular}{|c|c|c|c|c|}
\hline \multirow{2}{*}{ Номер узла } & \multicolumn{2}{|c|}{ До размещения } & \multicolumn{2}{c|}{$\begin{array}{c}\text { После размещения } \\
\text { конденсаторных батарей }\end{array}$} \\
\cline { 2 - 5 } & $\mathrm{U}, \%$ & $\cos \varphi$ & $\mathrm{U}, \%$ & $\cos \varphi$ \\
\hline 135 & 86,1 & 85 & 92,55 & 93,4 \\
\hline 136 & 86,6 & 90 & 91,695 & 93 \\
\hline 138 & 90,4 & 85 & 91,327 & 93 \\
\hline 139 & 91,3 & 85 & 91,818 & 93 \\
\hline 142 & 82,04 & 90 & 90,014 & 93 \\
\hline 144 & 82,6 & 90 & 92,528 & 93 \\
\hline 146 & 85,15 & 85 & 90,899 & 93,8 \\
\hline 148 & 84,8 & 85,4 & 91,066 & 90 \\
\hline 192 & 84,27 & 85,6 & 96,269 & 93 \\
\hline
\end{tabular}

чего наблюдается улучшение характеристики напряжения, уменьшение потерь и улучшение коэффициента мощности.

\section{Список литературы / References}

[1] Ahmed Z. Abass, D.A Pavlyuchenko,» The exploitation of western and southern deserts in Iraq for the production of solar energy» International Journal of Electrical and Computer Engineering (IJECE) Vol. 9, No. 6, December 2019, pp. 4617 4624 ISSN: 2088-8708, DOI: 10.11591/ijece.v9i6.pp4617-4624.

[2] Ahmed Z. Abass, D.A Pavlyuchenko,» Southern Iraq gas station conversation to integrated solar combined cycle», E3S web conferences 114, 05008 (2019) [Electronic resource] - Access: https://doi. org/10.1051/e3sconf/201911405008.

[3] Iraq, The Iraqi Ministry of Electricity, [Electronic resource] - Access: https://moelc.gov.iq/.

[4] Ahmed Z. Abass, D.A Pavlyuchenko,» Turning Iraq into a country of energy exporter through the exploitation of solar energy and vast desert land», E3S web conferences 114,05008 (2019) [Electronic resource] - Access: https://doi.org/10.1051/e3sconf/201911405008.

[5] Electrical Transient Analyzer Program (ETAP), [Electronic resource] - Access: www.etap.com.

[6] Kriti Singhal, «Comparison between Load Flow Analysis Methods in Power System using MATLAB», International Journal of Scientific \& Engineering Research, Volume 5, Issue 5, May 2014.

[7] Hadi Saadat, Power System Analysis, McGraw-Hill, 2006, 720 p.

[8] Muhammad A.U, Qamar Saeed, Abdul R A, Arslan Qaiser,» Load flow, voltage stability \& short circuit analyses and remedies for a $1240 \mathrm{MW}$ combined cycle power plant using ETAP» Conference: 2017 International Conference on Innovations in Electrical Engineering and Computational Technologies (ICIEECT) DOI: 10.1109/ICIEECT.2017.7916568.

[9] Kanchana Baby, K.L. Sreekumar,» Load Flow Analysis of 66 kV substation using ETAP Software» International Research Journal of Engineering and Technology (IRJET), V: 04 Issue: 02 | Feb -2017.

[10] Абасс А. 3., Павлюченко Д. А., Балабанов А. М., Лесс В. М. Включение в парогазовый цикл газотурбинных электростанций Ирака солнечной энергии как способ решения дефицита 
мощности энергосистемы страны. Известия высших учебных заведений. Проблемы энергетики. 2020;22(2):98-107 [Abass A.Z., Pavlyuchenko D. A., Balabanov A. M., Less V. M. Inclusion of solar energy in iraq gas-turbine power plants as a method of solving the country's energy system shortage. Power engineering: research, equipment, technology. 2020;22(2):98-107. (In Russ.)]

[11] A. Z. Abass; D. A. Pavlyuchenko; Zozan Saadallah Hussain, «Methods Comparison for Optimal Capacitor Placement in Distribution System», 2020 International Multi-Conference on Industrial Engineering and Modern Technologies (FarEastCon), 6-9 Oct. 2020, DOI: 10.1109/ FarEastCon50210.2020.9271381.

[12] A. H. A. Al-Waeli, M. M. El-Din, A.H. Al-Kabi, A. Al-Mamari, H. A. Kazem, and M. T. Chaichan, «Optimum design and evaluation of solar water pumping system for rural areas,» International Journal of Renewable Energy Research, vol.7, no. 1, pp. 12-20, 2017.

[13] Solar Learning Center, [Electronic resource] - Access: https://www.solar.com/learn/.

[14] Kehinde, O., A. S. Oluwole, T. Adefarati, «A fault analysis of $11 \mathrm{kV}$ distribution system (a case study of ado Ekiti electrical power distribution district),» American journal of electrical power and Energy system, volume 3, Issue 2, pages 22-36, 2014.

[15] Pushp raj, «Load flow and short circuit analysis of 400/220kv substation», International Journal of Creative and Research thoughts, vol. 1, Issue 4, April 2013.

[16] Muhammad Aman Ullah \& Abdul Rehman Abbasi, «Load Flow, Voltage Stability \& Short Circuit Analyses and Remedies for A 1240 MW Combined Cycle Power Plant Using ETAP» IEEE Trans. Power Syst., vol.10, no.1109, ICIEECT.2017.7916568, 5-7 April 2017.

[17] Ahmed Z. Abass, D.A Pavlyuchenko, Zozan Saadallah Hussain, «Survey about impact voltage instability and transient stability for a power system with an integrated solar combined cycle plant in Iraq by using ETAP,» Journal of Robotics and Control (JRC), Vol 2, No 3 (2021): May.

[18] Prabhu A, Priyadarshini S.A,» Design of Electrical System Using Mi Power on Load Flow and Stability Analysis», Imperial Journal of Interdisciplinary Research (IJIR), Vol. 3, Issue 122017. 